\title{
Analysis of microstructural effects in multi-layer lithium-ion battery cathodes
}

\author{
Daniel Westhoff ${ }^{\mathrm{a}, *}$, Timo Danner ${ }^{\mathrm{b}, \mathrm{c}}$, Simon Hein ${ }^{\mathrm{b}, \mathrm{c}}$, Rares Scurtu ${ }^{\mathrm{d}}$, \\ Lea Kremer ${ }^{\mathrm{d}}$, Alice Hoffmann ${ }^{\mathrm{d}}$, André Hilger ${ }^{\mathrm{e}}$, Ingo Manke ${ }^{\mathrm{e}}$, \\ Margret Wohlfahrt-Mehrens ${ }^{\mathrm{d}}$, Arnulf Latz ${ }^{\mathrm{b}, \mathrm{c}, \mathrm{f}}$, Volker Schmidt ${ }^{\mathrm{a}}$ \\ ${ }^{a}$ Ulm University, Institute of Stochastics, Helmholtzstraße 18, 89069 Ulm, Germany \\ ${ }^{b}$ Helmholtz Institute for Electrochemical Energy Storage (HIU), Helmholtzstraße 11, 89081 \\ Ulm, Germany \\ ${ }^{c}$ German Aerospace Center (DLR), Institute of Engineering Thermodynamics, \\ Pfaffenwaldring 38-40,70569 Stuttgart, Germany \\ ${ }^{d} Z S W$-Zentrum für Sonnenenergie- und Wasserstoff-Forschung Baden-Württemberg, \\ Helmholtzstraße 8,89081 Ulm, Germany \\ ${ }^{e}$ Helmholtz-Zentrum Berlin, Institute of Applied Materials, Hahn-Meitner-Platz 1, 14109 \\ Berlin, Germany \\ ${ }^{f}$ Ulm University, Institute of Electrochemistry, Albert-Einstein-Allee 47, 89081 Ulm, \\ Germany
}

\begin{abstract}
A possible way to increase the energy density in lithium-ion batteries, and, at the same time, reduce the production costs, is to use thicker electrodes. However, transport limitations can occur in thick electrodes, leading to a drawback in performance. A way to mitigate this problem is a more sophisticated microstructure of the electrode, using, e.g., structural gradients. This can, for instance, be achieved by multi-layer casting, i.e., casting and drying of a first layer, and then adding a second layer. An important question is how the interface between the two layers is shaped and how the corresponding microstructure influences the electrochemical performance. In the present paper, two different two-layer cathodes are analyzed and compared to single-layer cathodes of the same thickness. The analysis involved tomographic imaging, a statistical analysis of the $3 \mathrm{D}$ microstructure of the active material particle systems with a focus on the interface between the layers, and electrochemical characterization of the active material systems using experimental measurements as well as electrochemical simulations. The analysis showed that at the interface the connectivity of active material particles decreases, which results in higher electric resistivity. This ef-
\end{abstract}

\footnotetext{
* Corresponding author

Email address: daniel.westhoff@uni-ulm.de (Daniel Westhoff)
} 
fect is stronger if an intermediate calendering step is performed, i.e., the first layer is calendered before casting the second layer.

Keywords: lithium-ion batteries, multi-layer electrodes, microstructure, tomography, image analysis

\section{Introduction}

Because of their high energy density, lithium-ion batteries are widely used for energy storage in various applications ranging from mobile devices to electric vehicles [1. However, for a breakthrough in the automotive sector, the energy 5 density is still too small while processing costs are high [2. A way to increase the energy density while lowering the production costs at the same time is to use thicker electrodes, see [3] and the references therein. On the other hand, thicker electrodes can lead to charge transport limitations due to longer transport pathways 4. As it is well-known that the microstructure of electrodes strongly influences their performance (see, e.g., [5]), a possible way to overcome this problem is to use more sophisticated electrode microstructures, which, on the one hand, have high energy densities due to a high volume fraction of active material, but, on the other hand, provide sufficient transport pathways 6 . One method that can be used to achieve such structures is multi-layer casting, 15 where, after casting and drying of a first layer, a second layer is added, see 7]. Modern tomographic imaging methods render it possible to analyze the microstructure of battery electrodes in detail [8] and even in operando [9], which is an important input for modeling approaches. Several modeling papers review the potential of graded porosity in Li-ion battery electrodes [10, 11] but only moderate improvements in capacity and energy density were reported. However, it was shown that graded electrodes are able to produce a more homogeneous potential distribution which is supposed to reduce the degradation of the electrode [12, 13]. In [14, it is shown how a two-layer cathode using different active materials improves the safety of the battery.

A possible tool for further investigations of the electrochemical properties of multi-layer electrodes is to use stochastic 3D microstructure modeling [15] in combination with spatially resolved transport models [16, as it has for example been done to investigate the detrimental effects of the so-called Li plating 
in conventional graphite negative electrodes [17. A stochastic microstructure model for single-layer electrodes has, e.g., been developed in 18. In principle, it is possible to generate virtual multi-layer structures with such a model, which, e.g., exhibit a different particle size distribution in the first and second layer, respectively. However, also the microstructure at the interface between the individual layers has to be taken into account. Thus, a microstructural anal35 ysis of the interface as well as an investigation of its effects on electrochemical properties of the electrode is in the focus of the present paper.

Two different two-layer cathodes as well as two single-layer cathodes with different densities were considered. The first two-layer cathode was processed without individual calendering of the first layer, while intermediate calendering 40 of the first layer was performed for the second cathode before casting the second layer, see 19 for effects of calendering on the resulting electrodes. Note that, as the focus of the present paper is on the effect of the interface between the two layers, but not on how to design the individual layers to achieve an optimal performance, no further variation of the design of the top and the bottom layer 45 were performed. Electrochemical characterization of the active material was performed and 3D reconstructions were gained using tomographic imaging. By the aid of image analysis, the microstructural effects on the interface between the layers were analyzed and compared to apparent electronic resistivity gained by experiments. It turns out that, at the interface between the layers, particle 50 sizes as well as their connectivity are decreasing, which is in accordance with the higher apparent electronic resistivity (compared to the single-layer electrodes) observed in experiments.

The paper is organized as follows. In Section 2.1, the preparation of singlelayer and two-layer electrodes is described. Electrochemical characterization is described in Section 2.2. A description of the measurement of apparent electronic resistivity is provided in Section 2.3 Details on tomographic imaging are given in Section 3.1. and further processing steps of the image data are explained in Section 3.2. The analysis of image data is described in Section 3.3 . Conclusions are drawn in Section 4 . 
2. Performance analysis of single- and multi-layer cathodes

\subsection{Manufacturing of electrodes}

Positive electrodes were prepared by dispersing the active material $\mathrm{Li}\left[\mathrm{Ni}_{1 / 3} \mathrm{Co}_{1 / 3} \mathrm{Mn}_{1 / 3}\right] \mathrm{O}_{2}$ (NCM 111, Toda America), hereafter called NCM, conductive graphite additive (SFG6L, Timcal), and carbon black (SuperPLi, Tim-

65 cal) into a solution of polyvinylidene fluoride binder (Solef P5130, Solvay Solexis) in N-Methyl-2-pyrrolidone (NMP, Honeywell). The ratio of NCM, graphite, carbon black and binder was $91: 2.5: 2.5: 4$ by weight. Note that this ratio is approximately in accordance with common literature values, see [20]. We increased the ratio of NCM due to the desired application in high energy cells. Two different types of carbon have been used to combine their different advantageous properties [21, 22], see also [23] for methods to investigate the distribution of carbon black in the electrode.

The resulting suspension was cast in one or two steps onto an aluminum foil $(20 \mu \mathrm{m}, \mathrm{Korff})$ to result in single-layer or two-layer composite electrodes, respectively. After casting each layer, the corresponding sample was dried at $60^{\circ} \mathrm{C}$ for two hours and subsequently at $80^{\circ} \mathrm{C}$ overnight to evaporate the solvent. The electrode composites were densified using a calender (Summit) at $100^{\circ} \mathrm{C}$.

Four differently structured samples of electrodes were prepared, a two-layer electrode with one final calendering step (sample 1), a two-layer electrode with ${ }_{80}$ an intermediate and a final calendering step (sample 2), a single-layer electrode with low density (sample 3) and a single-layer electrode with medium density (sample 4). The mass loading of each electrode ranges between 19 and 21 $\mathrm{mg} / \mathrm{cm}^{2}$, which corresponds to the upper range of commercially relevant mass loadings used in state of the art lithium-ion cells. Circular blanks with an area 85 of $1.131 \mathrm{~cm}^{2}$ were punched from the samples to determine thickness and mass loading of the electrodes. Thickness of the electrodes and the aluminum foil were measured with a micrometer screw (Mitutoyo). Mass loading was determined by dividing the mass of the blanks of each electrode reduced by the mass of blanks of the aluminum foil by the area of the blanks. For the two-layer composite electrodes, measured values of mass were collected after the preparation of the first layer to obtain the mass loading of the first layer and after preparation of the second layer to obtain the mass loading of the overall composite, respectively. 
sample 1

\begin{tabular}{lrrrr}
\hline number of layers & 2 & 2 & 1 & 1 \\
intermediate calendering & no & yes & no & no \\
thickness whole composite in $\mu \mathrm{m}$ & 73.2 & 79.8 & 72.8 & 73.2 \\
mass loading layer $1 \mathrm{in} \mathrm{mg} / \mathrm{cm}^{2}$ & 6.5 & 8.08 & & \\
mass loading whole composite in $\mathrm{mg} / \mathrm{cm}^{2}$ & 18.9 & 19.9 & 19.5 & 21.2 \\
overall density of composite in $\mathrm{g} / \mathrm{cm}^{3}$ & 2.58 & 2.49 & 2.67 & 2.89
\end{tabular}

Table 1: Properties of the four samples of single-layer and two-layer electrodes.

The overall density of the electrode composite of each sample was calculated from their mass loading and thickness, respectively. Each value was determined 95 from the average of at least four measured values. An overview of the samples' properties is given in Table 1 .

\subsection{Electrochemical characterization}

The performance of final battery electrodes is mainly determined by their microstructure and the electrochemical properties of the active material. In order to provide a holistic description of the material system, in this section we address the electrochemical characterization of the active material. Furthermore, the electrochemical information in combination with the tomographic image data can be used as input for microstructure resolved simulations on the electrode scale which is a versatile tool for the development of novel electrode designs.

Open circuit voltage (OCV) measurements were performed using Swageloktype cells in a three electrode arrangement. Thin electrodes with a thickness of the composite of $18 \mu \mathrm{m}$ were used in this study in order to reduce mass transport limitations in the electrolyte. Preparation of these electrodes was performed analogously to the procedure described in Section 2.1. Cells were assembled in an argon-filled glove box, metallic lithium (Alfa Aesar) was used as the counter and the reference electrode, respectively. A piece of glass microfiber (Whatman, GF/A) was used as separator and $1 \mathrm{M} \mathrm{LiPF}_{6}$ in EC/EMC in the weight ratio of 3:7 with $2 \mathrm{wt} \% \mathrm{VC}$ (UBE Industries, Japan) was used 115 as electrolyte. Electrochemical data was collected at $25^{\circ} \mathrm{C}$ using a BaSyTec 

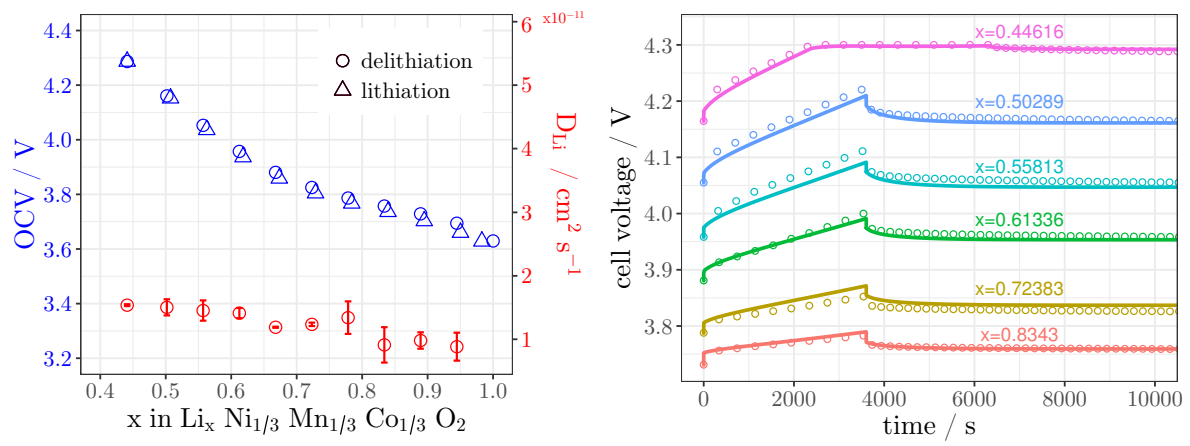

Figure 1: Left: OCV and chemical diffusion coefficient of lithium in NCM determined by constant-current \& potential-relaxation experiments. Right: Corresponding characteristic voltage response for delithiation experiments including simulations of the experimental setup. Dots show experimental values, lines show the corresponding simulations.

workstation, where all given potentials are referred to $\mathrm{Li} / \mathrm{Li}^{+}$. The cells were discharged to a cut-off voltage of $3.0 \mathrm{~V}$ with a constant current rate of $\mathrm{C} / 10$ and rested for $2 \mathrm{~h}$ to obtain the OCV related to a state of charge (SOC) of $0 \%\left(x \approx 1\right.$ in $\left.\mathrm{Li}_{x}\left[\mathrm{Ni}_{1 / 3} \mathrm{Co}_{1 / 3} \mathrm{Mn}_{1 / 3}\right] \mathrm{O}_{2}\right)$, with $\mathrm{SOC}=Q_{\text {res }} / Q_{\max }$, in which $Q_{\text {res }}$ represents residual capacity of the cathode, and $Q_{\max }$ is the maximum available capacity obtained with a current rate of $\mathrm{C} / 10$. Then the battery was charged in steps of $10 \% \mathrm{SOC}$ at a C-rate of $\mathrm{C} / 10$, each time followed by a relaxation period of $2 \mathrm{~h}$ to gain the respective OCV until a cut-off voltage of $4.3 \mathrm{~V}$ was reached, which corresponds to $\mathrm{SOC}=100 \%\left(x \approx 0.45\right.$ in $\left.\mathrm{Li}_{x}\left[\mathrm{Ni}_{1 / 3} \mathrm{Co}_{1 / 3} \mathrm{Mn}_{1 / 3}\right] \mathrm{O}_{2}\right)$. The near zero slope of the voltage signal at the end of each step (Figure 1, right) indicates that all processes in the cell are close to equilibrium after the 2 hours rest time. The same procedure was repeated for stepwise discharging and from this data, values of OCV as a function of SOC were collected for lithiation and delithiation. Figure 1, left, shows the corresponding OCV curve as a function of Li content in NCM. In our measurements we observed a minor hysteresis between lithiation and delithiation of the material which exceeds the standard deviation of $1 \mathrm{mV}$ determined on two different samples. The total capacity of the material during one lithiation and delithiation experiment in the voltage window between 4.3 and $3.6 \mathrm{~V}$ was $155.6 \mathrm{mAh} / \mathrm{g}$. Furthermore, the characteristic voltage relaxation signal provides additional information on the transport processes in the cell. The chemical diffusion of Li which equilibrates the concen- 
tration distribution on the particle scale determines the potential relaxation at long rest times. The particle size distribution was determined by laser diffraction on a Mastersizer MICRO analyzer from Malvern Instruments Ltd, where we used the medium particle diameter $\mathrm{D}_{50}=10.18 \mu \mathrm{m}$ to determine the chemical diffusion coefficient of $\mathrm{Li}$ in $\mathrm{NCM}$ following the methods proposed for the Galvanostatic Intermittent Titration Technique (GITT) [24, 25]. The resulting chemical diffusion coefficient of Li in NCM is shown in Figure 1, left. The results are in agreement with diffusion coefficients measured by GITT on similar NCM materials [26]. Finally, we determined the mean kinetic constant $i_{00}=3.34$. $10^{-7} \mathrm{~A} \mathrm{~m}^{2.5} \mathrm{~mol}^{-1.5}$ of the de-/intercalation reaction by fitting a pseudo-2D (P2D) continuum model [27] to the first $100 \mathrm{~s}$ of the constant-current phase measurements. In this initial stage concentration gradients in the electrolyte and active material are still minor and the main processes affecting the voltage response of the electrode are ohmic losses in the electrode and electrolyte as well as the activation overpotential of the intercalation reaction. Estimates for the conductivity of the electrode and electrolyte are provided by the measurements stated in Section 2.3 and data published in the literature [28. A brief description of the P2D model along with simulation parameters is given in Appendix A. The full voltage signal of representative constant-current and potential-relaxation cycles during a delithiation measurement are shown in Figure 1. right. The results of simulations which are parameterized based on the morphological parameters of the active material powder $\left(D_{50}\right)$ and the electrochemical data extracted from the measurements as described above (OCV, $\left.D_{\mathrm{Li}}, i_{00}\right)$ are in reasonable agreement with the measurements during both the constant-current and potential relaxation phase. This indicates a reliable determination of the key physical parameters which are needed as input for advanced simulations on a microstructural level.

\subsection{Analysis of apparent electronic resistivity}

Apparent electronic resistivity of the dry electrodes was measured by using an in-house developed experimental setup. The method is able to measure the electronic resistivity along the cross-section of an electrode [29, 30]. The experimental setup is shown in Figure 2 The measurement was made in an argon filled glove box to eliminate the influence of humidity on electrical propri- 


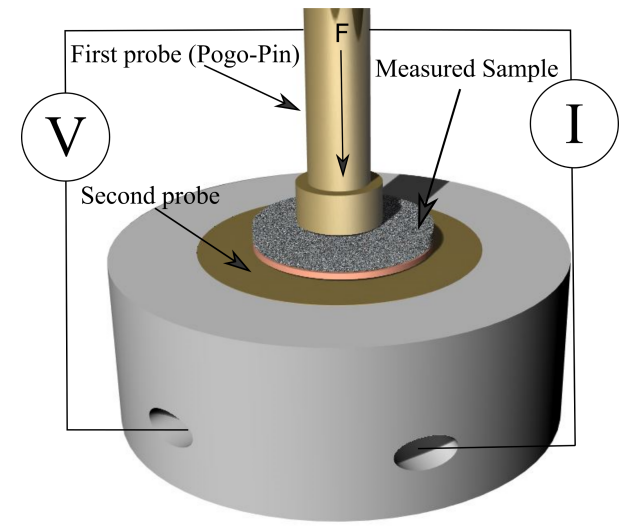

Figure 2: Experimental setup used for measuring the electrodes' electronic resistance.

eties of the electrodes. A disk shaped electrode with a diameter of $12 \mathrm{~mm}$ was placed between two gold coated probes and pressed with a constant force. The lower probe had a diameter of $2 \mathrm{~cm}$ and the upper probe was a pogo-pin with a diameter of $6.5 \mathrm{~mm}$. A plane-parallel alignment of the probes was achieved by the spring of the pogo-pin. The difference in diameters of the probes was eliminating the need to very carefully center the electrode.

A sourcemeter (Keithley Instruments Model 2400) with four wires arrangement was used to supply the direct current $I$ and to measure the voltage drop $U$ on the sample. The apparent resistivity $\rho$ was calculated via

$$
\rho=\frac{R \cdot A}{t},
$$

where $R=U / I$ is the calculated resistance, $A$ is the apparent contact area corresponding to the area of the upper probe and $t$ is the effective electrode coating thickness. The absolute value of the electrodes' resistivity cannot be measured with this method, because it cannot eliminate the influence of contact resistance at the probe/electrode interface. Still, it allows for a qualitative comparison of the different electrode samples.

The apparent resistivity of the electrodes is shown in Figure 3 . The error bars represent one standard deviation. Three different measured values were taken for each electrode. Note that the higher standard deviation of the electronic resistance measurements in case of sample 1 is most probably arising from its inhomogeneous compressibility due to its preparation (no intermediate calendaring). The electrode compressibility is an important parameter of the 


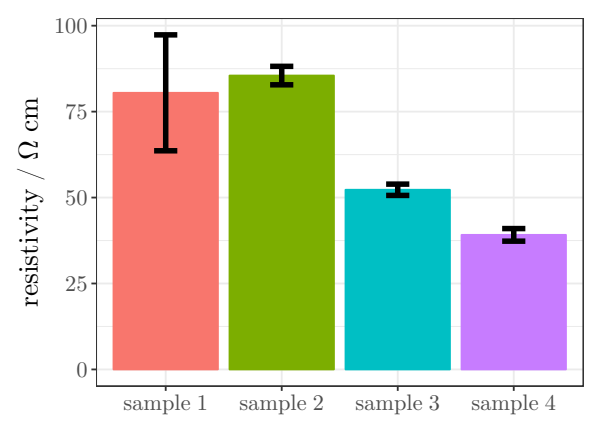

Figure 3: Apparent resistivity of the electrodes.

$\mathrm{LiB}$ electrodes and should be a topic of future investigations. Even with the higher standard deviation, the resistivity of sample 1 is comparable with sample 2 value and signicant higher than resistivity of sample 3 and sample 4. Thus, the single-layer electrodes show a lower resistivity in comparison with the multilayer electrodes. These differences can be explained by contact resistance of the interface between the layers. In Section 3.3 , structural characteristic that can lead to such a contact resistance are discussed in detail.

\section{Microstructural image analysis}

\subsection{Tomographic imaging}

In order to analyze microstructural properties of the active material particle system (which we will shortly denote by "active particle system" in the following), tomographic imaging was performed at the synchroton X-ray facility BAMLine (BESSY, Berlin, Germany). A Si-W multilayer monochromator was used to produce a monochromatic X-ray beam with an energy of $25 \mathrm{keV}$ and an energy resolution of $\Delta \mathrm{E} / \mathrm{E}=10^{-2}$. A cadmium tungstate scintillator screen converted the X-rays into visible light. The optical lens system in combination with a CCD-camera (PCO camera, $4008 \times 2672 \mathrm{~mm}^{2}$ ) covered a field of view of $1.8 \times 1.2 \mathrm{~mm}^{2}$. The pixel size of the system was $446 \mathrm{~nm}$ in all dimensions. The exposure time was 2.5 seconds and over an angular range of $180^{\circ}$ a number of 2200 projections were measured. The procedure resulted in a 16-bit grayscale image for each of the four samples. 


\subsection{Processing of image data} narization, i.e., to convert the grayscale images to binary images showing the active particle phase. Note that the conductive graphite, carbon black and binder are not visible in the grayscale images due to the low contrast of the corresponding materials. In 31, it was found that the distribution of binder for the effects of tortuosity. Thus, the further structural analysis focuses on properties of the active particle system, and structural characteristics of this system are compared to the electronic resistivity. Note that conductivity in lithium-ion battery cathodes is strongly influenced by carbon black. However, this additive is typically located around the particles 33 . Therefore, it is reasonable to assume that the arrangement of particles and their connectivity play an important role for electronic conductivity / resistivity.

From the weight ratio of the materials given in Section 2.1 and the corresponding densities as well as the density of the whole electrode, we can compute

The images were binarized by global thresholding, i.e., every voxel the gray value of which is greater than or equal to some threshold $\tau$ was assigned to the particle phase, and the remaining voxels were assigned to the complementary phase. For sample 4, a volume fraction of the active particle phase of $57.1 \%$ was ing a sufficiently large cutout of the grayscale image, a value of $\tau=41129$ was found to match this volume fraction. A comparison of a cutout of a $2 \mathrm{D}$ slice from the grayscale image stack of sample 4 with the corresponding binarization can be found in Figure 4. The same threshold was then used to binarize all four images.

A problem for further processing and analysis of the data is that the layers can be slightly twisted, see Figure 5, top. Therefore, the layers were straightened by fitting a polynom of degree $5 \times 5$ to the particle voxels on the bottom of the layer, and shifting each bottom voxel down by the distance to the minimum

bottom. From this visualization, it can be seen that the spatial arrangement 


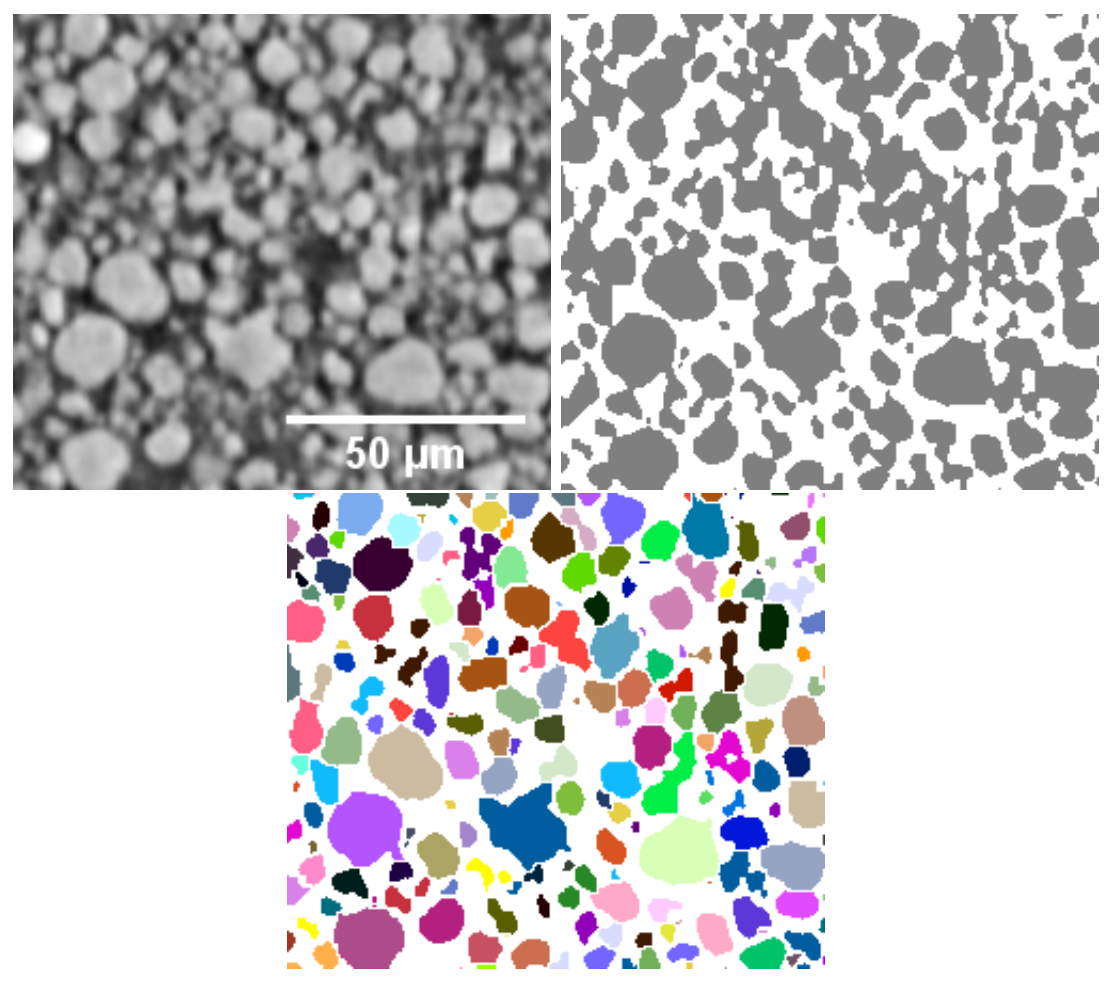

Figure 4: Top left: Cutout of a slice of the grayscale image of sample 4. Top right: Binarization, where gray color corresponds to the active particle phase. Bottom: Segmentation of individual particles labeled in different colors. 


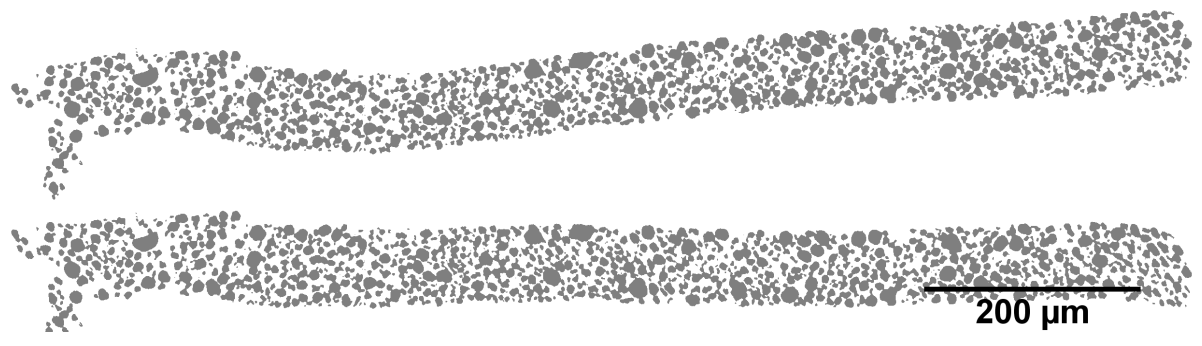

Figure 5: Top: 2D cross-section of sample 1 after binarization. Bottom: Same slice after straightening of the layer.

of particles to each other is not disturbed, but the whole layer is straightened. Additionally, it has been checked that no structural changes occur by comparing image characteristics of a reasonable cutout of the unstraightened layer with those of cutouts from the straightened layer.

For each of the four samples, we took three distinct cutouts out of the whole stack. These cutouts were used for further analysis. Each cutout has a horizontal extension of $500 \times 500$ voxels, corresponding to $223 \times 223 \mu \mathrm{m}$. To facilitate further processing, holes within particles were closed using a cluster detection algorithm [34.

In order to investigate particle-based characteristics, a segmentation of individual particles was performed. This is typically done using the watershed algorithm, see [35] for an overview. We used an extended version that has been proposed in [36], with an additional postprocessing that has been introduced in 37 to remove small segments induced by oversegmentation. Moreover, particles with a size of less than 9 voxels are considered as artefacts and removed. A segmented cutout from sample 1 is shown in Figure 6, top. A comparison of a 2D slice with the corresponding grayscale image is provided in Figure 4 , right. As supplementary information, a comparison of a whole grayscale image stack with the corresponding segmentation is provided.

Finally, for the analysis of the active particle systems, a so-called connectivity graph was extracted from each segmented image. The connectivity graph contains the particles' barycenters as nodes, and an edge was put between two nodes if the corresponding particles are in contact. A visualization of the graph can be found in Figure 6, bottom. 

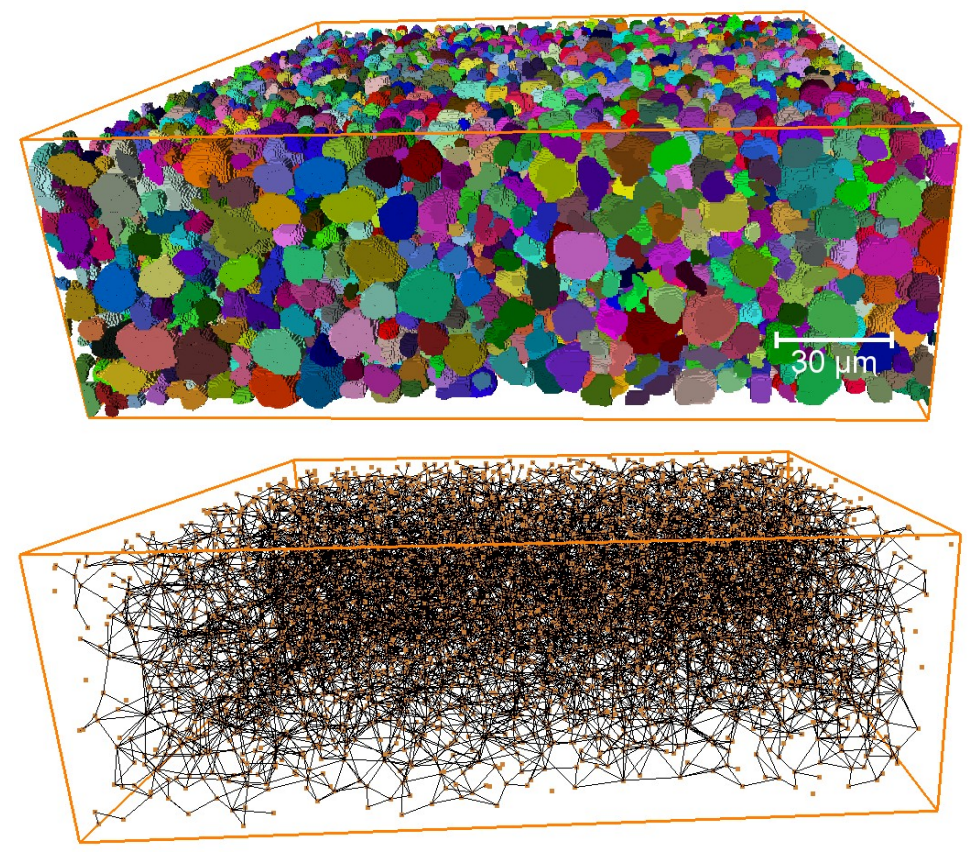

Figure 6: Cutout of sample 1. Top: Active particle phase segmented into individual particles (shown in different colors). Bottom: Connectivity graph of the particle system. Black lines connect centers of particles (orange spheres) which are in contact. 


\subsection{Analysis of morphological properties}

The preprocessing steps described in Section 3.2 resulted in three segmented cutouts for each of the four samples as well as corresponding graphs describing connectivity of particles. To begin with, we compared the volume fraction of the active particle phase of the segmented images to the one that has been calculated from the weight ratio of the materials that is known from the manufacturing process. In Figure 7, top left, it can be observed that for sample 4, which has been used for fitting the threshold $\tau$, the volume fraction of the active particle phase in the three cutouts approximately matches the one calculated from the weight ratio of the materials. For sample 3, the volume fraction is slightly larger than the one calculated from the weight ratio of the materials, however, for samples 1 and 2, on which the further analysis mainly focuses, again a quite good accordance can be observed.

An important characteristic for the analysis of local effects at the interface between the two layers in sample 1 and 2 is the local volume fraction in dependence of the distance to the current collector foil. Therefore, we computed the volume fraction of each slice of the image stack. This characteristic is prone to boundary effects, because the top and bottom of the electrode are not totally smooth, leading to smaller local volume fractions at the boundaries. Therefore, the first slice we considered for each cutout is the one where the overall volume fraction of the active particle phase has reached up to $0.005 \%$. In order to enhance comparability between different cutouts and samples, we define this slice to have zero distance to the bottom of the electrode, and computed the distance to this slice for the further slices. We did not consider slices any more after the one where the considered volume fraction is reached the last time when going through the stack. The results can be found in Figure 7, top right. The shaded areas show the (pointwise calculated) standard deviations, solid lines show the mean values per sample. We find that the local volume fraction is almost constant for samples 3 and 4, however, for samples 1 and 2, a decrease of the volume fraction can be observed approximately $25 \mu \mathrm{m}$ after the first considered slice of each stack. This effect is more pronounced for sample 2, where an intermediate calendering step was performed before casting the second layer. The observed decrease in volume fraction at the interface corresponds to the local distribution 

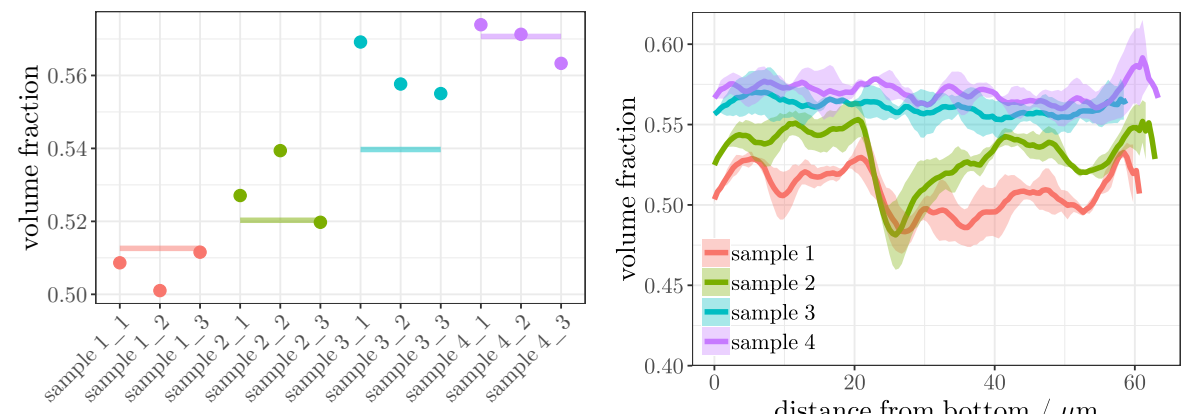

distance from bottom $/ \mu \mathrm{m}$
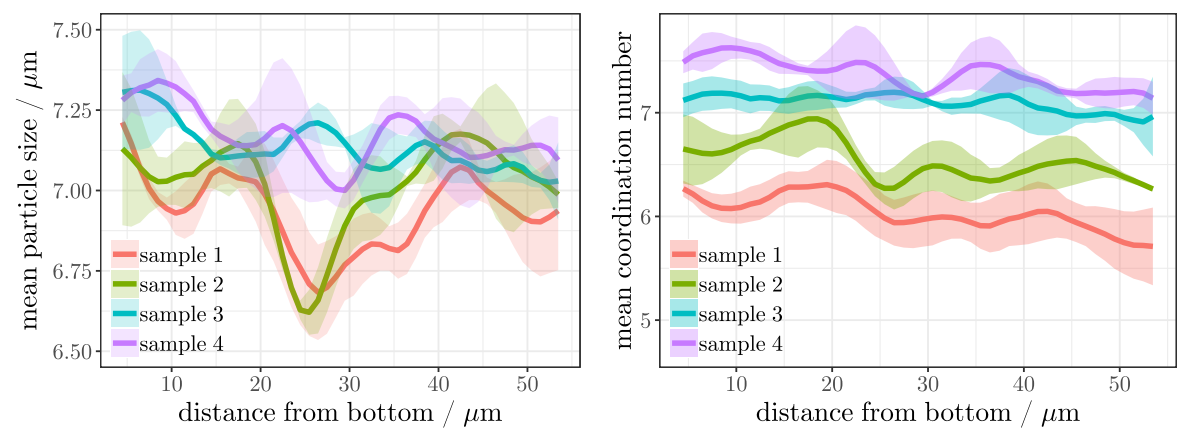

Figure 7: Structural characteristics of the active particle systems in the four different electrodes: Top left: Volume fraction of active particle phase. Dots show results for the three cutouts per sample, lines show the computed volume fraction from weight ratios of the corresponding materials. Top right: Local volume fraction in dependence of the distance to the collector foil. Bottom left: Mean particle size in dependence of the distance to the collector foil. Bottom right: Mean number of adjacent particles per particle in dependence of the distance to the collector foil. The shaded areas show show the pointwise standard deviations of the curves, solid lines show the mean values per sample. The origin of the $\mathrm{x}$-axis has been shifted for each cutout as described in Section 3.3 to enhance comparability. 
of particle sizes, see Figure 7, bottom left. Here, the mean particle size is shown in dependence of the distance to the first slice, where the $\mathrm{x}$-axis is shifted by the same value for each cutout as for the local volume fraction. The curves have been determined using kernel smoothing based on the Nadaraya-Watson estimator 38 with a bandwidth of $h=5 \mu \mathrm{m}$. Additionally, an edge correction has been performed to exclude particles that are not fully contained in the sampling window. We find that particle sizes seem to decrease slightly from bottom to top for samples 3 and 4, however, for samples 1 and 2, we observe distinctly smaller particle sizes at the interface between the two layers, where this effect is again more pronounced for the electrode with intermediate calendering. Note that both effects, the lower volume fraction at the interface and smaller particle sizes, can also be observed in the stack of grayscale images, which is given as supplementary information. Moreover, note that the oscillations of the curves also for single-layer electrodes are likely to be explained by the fact that approximately spherical particles are "packed" in a cubic sampling window. In 39, effects on the local volume fractions have been considered for packings of equally sized spheres in containers, and damping oscillations with increasing distance to the container wall are observed. For the battery electrodes under considerations, this effect is less pronounced because the particles are no perfect spheres and have different sizes, which leads to less regular oscillations. Moreover, the oscillations are smoothed by averaging over three samples. This is likely to explain the irregular osscilations of the curves in Figure 7. Finally, the connectedness of the particle systems was analyzed, which is a measure for the apparent electronic resistivity of the electrode samples. Using the connectivity graph, the extraction of which has been described in Section 3.2, we computed the number of particles which each particle is in contact with. We call this value the coordination number of the particle. A corresponding plot can be found in Figure 7 . bottom right, using the same shifting and bandwidth for kernel smoothing as described above. We find that the mean coordination number decreases slightly at the interface between two layers. Note that, in contrast to the mean particle size, this decrease is not compensated in the second layer, but the mean coordination number settles down after the decrease.

To summarize, we find that at the interface between two layers, the volume 
fraction of active material as well as the mean size of particles is smaller than within the two layers. This effect is more pronounced for the electrode with an intermediate calendering step, and in both cases ultimately leads to a loss in connectivity of the particle system. This is in agreement with the experimental results discussed in Section 2.3 . Thus, we conclude that the microstructural effects at the interface between the two layers have a strong influence on electrochemical properties, namely the apparent electronic resistivity.

\section{Conclusions and outlook}

Microstructural and electrochemical effects in multi-layer electrodes are investigated. Four different samples were prepared and electrochemically characterized. Tomographic images were gained, based on which a microstructural analysis was performed. The comparison with single-layer electrodes shows that in two-layer electrodes, microstructural effects at the interface between the two layers limit electrochemical processes. It turns out that the volume fraction of active material as well as average particle sizes and their connectivity with each other is decreasing, which, in turn, limits apparent electronic conductivity. Note that the main focus of the present paper is on the interface between the layers, and that different designs of the individual layers might compensate this negative effect at the interface, leading to an overall better performance of multi-layer electrodes.

From the analysis presented in this paper, it can be concluded that, when using multi-layer electrodes to improve electrode performance in lithium-ion batteries, special attention has to be paid to the interface between the layers and preparation techniques that limit negative structural features have to be developed. On the one hand, structural differences at the interface can be explained by mechanical reasons, i.e., the alignment of particles in the supsension that is casted on an already dried first layer. However, also the drying process itself might influence the distribution of binder, carbon black and conductive graphite. In future work, it would be interesting to focus on the spatial distribution of these additives, which are not visible using the tomographic imaging procedure described in the present paper. E.g., in [40, the usage of multi-layer electrodes with a different amount of binder in the slurry for the top layer than for the 
bottom layer resulted in a preferable binder distribution in the overall electrode.

Besides that, it is worth to analyze how different calendering procedures influence particle connectivity at the interface between the layers. Moreover, the results give valuable information on how to simulate the interface between the layers for model-based simulations of electrochemical properties based on the combination of stochastic 3D microstructure modeling with spatially resolved transport models, as it has e.g. been done in [41].

\section{Acknowledgements}

This work was partially funded by BMBF under grant numbers 03XP0073C, 03XP0073D and 03XP0073E.

\section{Data availability}

The raw/processed data required to reproduce these findings cannot be shared at this time as the data also forms part of an ongoing study.

\section{References}

\section{References}

[1] G.-A. Nazri, G. Pistoia (Eds.), Lithium Batteries: Science and Technology, Springer, New York, 2003.

[2] M. M. Thackeray, C. Wolverton, E. D. Isaacs, Electrical energy storage for transportation - approaching the limits of, and going beyond, lithiumion batteries, Energy \& Environmental Science 5 (7) (2012) 7854-7863. doi:10.1039/C2EE21892E

[3] M. Singh, J. Kaiser, H. Hahn, Thick electrodes for high energy lithium ion batteries, Journal of The Electrochemical Society 162 (7) (2015) A1196A1201. doi:10.1149/2.0401507jes

[4] T. Danner, M. Singh, S. Hein, J. Kaiser, H. Hahn, A. Latz, Thick electrodes for li-ion batteries: A model based analysis, Journal of Power Sources 334 (2016) 191-201. doi:10.1016/j.jpowsour.2016.09.143. 
[5] J. R. Wilson, J. S. Cronin, S. A. Barnett, S. J. Harris, Measurement of three-dimensional microstructure in a $\mathrm{LiCoO}_{2}$ positive electrode, Journal of Power Sources 196 (7) (2011) 3443-3447. doi:10.1016/j.jpowsour. 2010.04 .066

[6] S. Behr, R. Amin, Y. M. Chiang, A. P. Tomsia, Highly-structured, additivefree lithium-ion cathodes by freeze-casting technology, Ceramic Forum International 92 (4) (2015) E39-E43.

[7] J. Whitacre, K. Zaghib, W. West, B. Ratnakumar, Dual active material composite cathode structures for li-ion batteries, Journal of Power Sources 177 (2) (2008) 528-536. doi:10.1016/j.jpowsour.2007.11.076.

[8] M. Ebner, F. Geldmacher, F. Marone, M. Stampanoni, V. Wood, X-ray tomography of porous, transition metal oxide based lithium ion battery electrodes, Advanced Energy Materials 3 (7) (2013) 845-850. doi:10. 1002/aenm. 201200932.

[9] D. P. Finegan, M. Scheel, J. B. Robinson, B. Tjaden, M. D. Michiel, G. Hinds, D. J. L. Brett, P. R. Shearing, Investigating lithium-ion battery materials during overcharge-induced thermal runaway: an operando and multi-scale X-ray CT study, Physical Chemistry Chemical Physics 18 (2016) 30912-30919.

[10] Y. Dai, V. Srinivasan, On graded electrode porosity as a design tool for improving the energy density of batteries, Journal of The Electrochemical Society 163 (3) (2016) A406-A416. doi:10.1149/2.0301603jes

[11] Z. Du, D. L. Wood, C. Daniel, S. Kalnaus, J. Li, Understanding limiting factors in thick electrode performance as applied to high energy density Liion batteries, Journal of Applied Electrochemistry 47 (3) (2017) 405-415. doi:10.1007/s10800-017-1047-4.

[12] L. Liu, P. Guan, C. Liu, Experimental and simulation investigations of porosity graded cathodes in mitigating battery degradation of high voltage lithium-ion batteries, Journal of The Electrochemical Society 164 (13)

${ }_{420}$ (2017) A3163-A3173. doi:10.1149/2.1021713jes 
[13] Y. Qi, T. Jang, V. Ramadesigan, D. T. Schwartz, V. R. Subramanian, Benefit analysis of employing graded electrodes in lithium-ion batteries, Journal of The Electrochemical Society 164 (13) (2017) A3196-A3207. doi : $10.1149 / 2.1051713$ jes

${ }_{425}$ [14] N. Imachi, Y. Takano, H. Fujimoto, Y. Kida, S. Fujitani, Layered cathode for improving safety of li-ion batteries, Journal of The Electrochemical Society 154 (5) (2007) A412-A416. doi:10.1149/1.2709503

[15] M. Neumann, V. Schmidt, Stochastic 3D modeling of amorphous microstructures - a powerful tool for virtual materials testing, Proceedings of the VII European Congress on Computational Methods in Applied Sciences and Engineering (2016) Paper-ID 8172doi:10.7712/100016.1922.8172.

[16] A. Latz, J. Zausch, Thermodynamic consistent transport theory of Li-ion batteries, Journal of Power Sources 196 (6) (2011) 3296-3302. doi:10. $1016 / j \cdot$ jpowsour.2010.11.088

[18] D. Westhoff, J. Feinauer, K. Kuchler, T. Mitsch, I. Manke, S. Hein, A. Latz, V. Schmidt, Parametric stochastic 3D model for the microstructure of anodes in lithium-ion power cells, Computational Materials Science 126 (2017) 453-467. doi:10.1016/j.commatsci.2016.09.006.

[19] H. Zheng, L. Tan, G. Liu, X. Song, V. S. Battaglia, Calendering effects on the physical and electrochemical properties of $\mathrm{Li}\left[\mathrm{Ni}_{1 / 3} \mathrm{Mn}_{1 / 3} \mathrm{Co}_{1 / 3}\right] \mathrm{O}_{2}$ cathode, Journal of Power Sources 208 (2012) 52-57. doi:10.1016/j . jpowsour.2012.02.001.

[20] D. Andre, S.-J. Kim, P. Lamp, S. F. Lux, F. Maglia, O. Paschos, B. Stiaszny, Future generations of cathode materials: an automotive industry 
perspective, Journal of Materials Chemistry A 3 (13) (2015) 6709-6732. doi:10.1039/C5TA00361J

[21] J. W. Fergus, Recent developments in cathode materials for lithium ion batteries, Journal of Power Sources 195 (4) (2010) 939-954. doi:10.1016/ j.jpowsour.2009.08.089.

[22] S. Mandal, J. M. Amarilla, J. Ibanez, J. M. Rojo, The role of carbon black in limn2o4-based composites as cathodes for rechargeable lithium batteries, Journal of The Electrochemical Society 148 (1) (2001) A24-A29. doi:10.1149/1.1339026.

[23] L. Pfaffmann, S. Jaiser, M. Müller, P. Scharfer, W. Schabel, W. Bauer, F. Scheiba, H. Ehrenberg, New method for binder and carbon black detection at nanometer scale in carbon electrodes for lithium ion batteries, Journal of Power Sources 363 (2017) 460-469. doi:10.1016/j.jpowsour. 2017.07.102.

[24] Q. Wang, H. Li, X. Huang, L. Chen, Determination of chemical diffusion coefficient of lithium ion in graphitized mesocarbon microbeads with potential relaxation technique, Journal of The Electrochemical Society 148 (7) (2001) A737. doi:10.1149/1.1377897.

[25] P. D. Weidman, D. Ahn, R. Raj, Diffusive relaxation of Li in particles of silicon oxycarbide measured by galvanostatic titrations, Journal of Power Sources 249 (2014) 219-230. doi:10.1016/j.jpowsour.2013.09.087.

[26] H.-J. Noh, S. Youn, C. S. Yoon, Y.-K. Sun, Comparison of the structural and electrochemical properties of layered $\mathrm{Li}\left[\mathrm{Ni}_{x} \mathrm{Co}_{y} \mathrm{Mn}_{z}\right] \mathrm{O}_{2}(\mathrm{x}=1 / 3,0.5$, 0.6, 0.7, 0.8 and 0.85) cathode material for lithium-ion batteries, Journal of Power Sources 233 (2013) 121-130. doi:10.1016/j.jpowsour.2013.01. 063 .

[27] M. Doyle, J. Newman, The use of mathematical modeling in the design of lithium/polymer battery systems, Electrochimica Acta 40 (13-14) (1995) 2191-2196. doi:10.1016/0013-4686(95)00162-8. 
[28] A. Nyman, M. Behm, G. Lindbergh, Electrochemical characterisation and modelling of the mass transport phenomena in $\mathrm{LiPF}_{6}-\mathrm{EC}-\mathrm{EMC}$ electrolyte, Electrochimica Acta 53 (22) (2008) 6356-6365. doi:10.1016/j. electacta.2008.04.023.

[29] M. Indrikova, S. Grunwald, F. Golks, A. Netz, B. Westphal, A. Kwade, The morphology of battery electrodes with the focus of the conductive additives paths, Journal of The Electrochemical Society 162 (10) (2015) A2021-A2025. doi:10.1149/2.0441510jes.

[30] B. G. Westphal, N. Mainusch, C. Meyer, W. Haselrieder, M. Indrikova, P. Titscher, H. Bockholt, W. Viöl, A. Kwade, Influence of high intensive dry mixing and calendering on relative electrode resistivity determined via an advanced two point approach, Journal of Energy Storage 11 (2017) 7685. doi:10.1016/j.est.2017.02.001

[31] J. Landesfeind, M. Ebner, A. Eldiven, V. Wood, H. A. Gasteiger, Tortuosity of battery electrodes: Validation of impedance-derived values and critical comparison with 3D tomography, Journal of The Electrochemical Society 165 (3) (2018) A469-A476. doi:10.1149/2.0231803jes.

[32] M. Ebner, D. W. Chung, R. E. García, V. Wood, Tortuosity anisotropy in lithium-ion battery electrodes, Advanced Energy Materials 4 (5) (2014) 1-6. doi:10.1002/aenm.201301278.

[33] M. Ender, J. Joos, T. Carraro, E. Ivers-Tiffée, Three-dimensional reconstruction of a composite cathode for lithium-ion cells, Electrochemistry Communications 13 (2) (2011) 166 - 168. doi:10.1016/j.elecom.2010. 12.004

[34] J. Hoshen, R. Kopelman, Percolation and cluster distribution. I. Cluster multiple labeling technique and critical concentration algorithm, Physical Review B 14 (8) (1976) 3438-3445. doi:10.1103/PhysRevB.14.3438.

[35] J. B. T. M. Roerdink, A. Meijster, The watershed transform: Definitions, algorithms and parallelization strategies, Fundamenta Informaticae 41 (2000) 187-228. doi:10.3233/FI-2000-411207. 

V. Schmidt, Stochastic 3D modeling of ostwald ripening at ultra-high volume fractions of the coarsening phase, Modelling and Simulation in [ Materials Science and Engineering 23 (6) (2015) 065001. doi:10.1088/ $0965-0393 / 23 / 6 / 065001$

[37] K. Kuchler, D. Westhoff, J. Feinauer, T. Mitsch, I. Manke, V. Schmidt, Stochastic model for the 3D microstructure of pristine and cyclically aged cathodes in Li-ion batteries, Modelling and Simulation in Materials Science and Enginnering 26 (2018) 035005. doi:10.1088/1361-651X/aaa6da.

[38] E. Nadaraya, Nonparametric Estimation of Probability Densities and Regression Curves, Kluwer, Dordrecht, 1989.

[39] R. F. Benenati, C. B. Brosilow, Void fraction distribution in beds of spheres, AIChE Journal 8 (3) (1962) 359-361.

[40] D. Liu, L.-C. Chen, T.-J. Liu, W.-B. Chu, C. Tiu, Improvement of lithiumion battery performance by two-layered slot-die coating operation, Energy Technology 5 (8) (2017) 1235-1241. doi:10.1002/ente.201600536

[41] S. Hein, J. Feinauer, D. Westhoff, I. Manke, V. Schmidt, A. Latz, Stochastic microstructure modeling and electrochemical simulation of lithium-ion cell anodes in 3D, Journal of Power Sources 336 (2016) 161-171. doi:10.1016/ j.jpowsour.2016.10.057.

[42] S. G. Meibuhr, Electrode studies in nonaqueous electrolytes, Journal of The Electrochemical Society 118 (1) (1970) 1320. doi:10.1149/1.2407438

\section{Appendix - P2D model}

In this work we use the well established P2D model derived by Doyle et al. 27] for a battery cell consisting of a porous electrode made of spherical particles with radius $R_{p}$, a porous separator, and a lithium metal anode. The purpose of this appendix is to summarize the governing equations and parameters. A detailed discussion and explanation can be found in the original research paper. 
In this work we additionally assume isothermal conditions and fix the temperature in the cell to $T=298 \mathrm{~K}$. In the P2D model the temporal evolution of the volume averaged lithium ion concentration $c_{\mathrm{e}}$ in the electrolyte is described by the mass balance of lithium ions

$$
\frac{\partial \varepsilon_{\mathrm{e}} c_{\mathrm{e}}}{\partial t}=-\frac{\partial}{\partial x}\left(-D_{\mathrm{e}} \varepsilon_{\mathrm{e}}^{\beta} \frac{\partial c_{\mathrm{e}}}{\partial x}+\frac{t_{\mathrm{Li}^{+}} i_{\mathrm{e}}}{F}\right)+\frac{a^{v} i_{s e}}{F},
$$

where $\varepsilon_{\mathrm{e}}$ is the electrolyte volume fraction, $D_{\mathrm{e}}$ the diffusion coefficient of lithium ions in the electrolyte, $\beta$ the Bruggeman coefficient as a measure for the tortuosity of the electrode, $t_{\mathrm{Li}^{+}}$the transference number of lithium ions, $i_{e}$ the ionic current in the electrolyte, $F$ the Faraday constant, $a^{v}=3 \varepsilon_{\mathrm{e}} / R_{p}$ the volume specific surface area, and $i_{s e}$ the Faradaic current of the intercalation reaction. The ionic current in the electrolyte is given by

$$
i_{\mathrm{e}}=-\kappa \varepsilon_{\mathrm{e}}^{\beta} \frac{\partial \phi_{\mathrm{e}}}{\partial x}-\kappa_{D} \varepsilon_{\mathrm{e}}^{\beta} \frac{\partial c_{\mathrm{e}}}{\partial x},
$$

with the ionic conductivity of the electrolyte $\kappa$, the electrochemical potential of lithium ions in the electrolyte $\phi_{\mathrm{e}}$ and the so-called diffusional conductivity

$$
\kappa_{D}=\frac{2 \kappa R T\left(t_{\mathrm{Li}^{+}}-1\right)}{F c_{\mathrm{e}}}\left(1+\frac{\partial \ln f_{\mathrm{Li}^{+}}}{\partial \ln c_{\mathrm{e}}}\right),
$$

where $1+\frac{\partial \ln f_{\mathrm{Li}^{+}}}{\partial \ln c_{\mathrm{e}}}$ is the so-called thermodynamic factor, and $R$ the universal gas constant. Based on the assumption of electro-neutrality charge conservation in the electrolyte follows as

$$
0=-\frac{\partial i_{\mathrm{e}}}{\partial x}+a^{v} i_{s e} .
$$

The second term on the right hand side describes the exchange of charge (\& also mass cf. Eq. (1) ) due to the de-/intercalation of lithium ions in NCM and the deposition on the Li metal electrode

$$
\mathrm{Li}^{+}+\mathrm{e}^{-} \rightleftharpoons \mathrm{Li}
$$

The reaction rate at the NCM positive electrode is described by the ButlerVolmer equation

$$
\begin{aligned}
i_{\mathrm{se}}^{\mathrm{ca}} & =i_{0}\left[\exp \left(\frac{\alpha F}{R T} \eta\right)-\exp \left(-\frac{(1-\alpha) F}{R T} \eta\right)\right] \\
& =i_{00}^{\mathrm{ca}} c_{\mathrm{e}}^{\alpha} c_{\mathrm{s}}^{\alpha}\left(c_{\mathrm{s}}^{\max }-c_{\mathrm{s}}\right)^{1-\alpha}\left[\exp \left(\frac{\alpha F}{R T} \eta\right)-\exp \left(-\frac{(1-\alpha) F}{R T} \eta\right)\right],
\end{aligned}
$$


where $i_{00}$ is the exchange-current density, $c_{\mathrm{s}}$ and $c_{\mathrm{s}}^{\max }$ are the local and maximum lithium concentration in the active material, $\alpha$ is the symmetry factor of the transition state, and $\eta=\phi_{s}-\phi_{e}-U_{0}\left(c_{\mathrm{s}} / c_{\mathrm{s}}^{\max }\right)$ the overpotential driving the electrochemical reaction. The open circuit voltage $U_{0}$ depends on the lithiation of the active material and was in this work determined to

$$
\begin{array}{r}
U_{0}(\mathrm{SOC})=5.2652-6.9654 \mathrm{SOC}+5.5261 \mathrm{SOC}^{1.2603}- \\
1.7756 \cdot 10^{-8} \exp (132.6405 \mathrm{SOC}+-114.2593)-0.1173 \mathrm{SOC}^{-0.4166} .
\end{array}
$$

At the lithium metal anode a constant exchange-current density of $1 \mathrm{~mA} / \mathrm{cm}^{2}$ 42 is assumed, reducing the expression of the deposition rate to

$$
i_{\mathrm{se}}^{\mathrm{an}}=2 i_{00}^{\mathrm{an}} \sinh \left(\frac{0.5 F}{R T}\left(\phi_{s}-\phi_{e}\right)\right) .
$$

The capacity of the cell is determined by the amount of lithium which can be stored in the active material. The de-/intercalation of lithium ions at the particle surface (Eq. (6) and transport in the particles by diffusion $D_{\mathrm{s}}$ is described by

$$
\frac{\partial c_{\mathrm{s}}}{\partial t}=-\frac{1}{r^{2}} \frac{\partial}{\partial r}\left(-r^{2} D_{\mathrm{s}} \frac{\partial c_{\mathrm{s}}}{\partial r}\right) .
$$

Transport of electrons in the solid electrode phase is modeled by Ohm's law

$$
0=-\frac{\partial i_{\mathrm{s}}}{\partial x}-a^{v} i_{s e}=-\frac{\partial}{\partial x}\left(-\sigma^{\mathrm{eff}} \frac{\partial \phi_{\mathrm{s}}}{\partial x}\right)-a^{v} i_{s e},
$$

where $\sigma^{\text {eff }}$ is the effective conductivity of the electrode. The equations are discretized in space by a finite volume method. The resulting system of differential algebraic equations is solved in Matlab using ode15s. All model parameters are summarized in Table 2 . 


\begin{tabular}{|c|c|c|}
\hline & $\mathrm{NCM}$ & Separator \\
\hline Electrolyte & \\
\hline$c_{\mathrm{e}}^{0} / \mathrm{mol} \mathrm{m}^{-3}$ & \multicolumn{2}{|l|}{1000 28] } \\
\hline$\kappa / \mathrm{S} \mathrm{m}^{-1}$ & \multicolumn{2}{|l|}{0.95128} \\
\hline$t_{\mathrm{Li}^{+}} /-$ & \multicolumn{2}{|l|}{0.25328} \\
\hline$D_{\mathrm{e}} / \mathrm{m}^{2} \mathrm{~s}^{-1}$ & \multicolumn{2}{|l|}{$3.76 \cdot 10^{-10} \quad 28$} \\
\hline$\left(1+\ln f_{e} / \partial \ln c_{e}\right) /-$ & \multicolumn{2}{|l|}{1.99 [28] } \\
\hline$\beta /-$ & 1.5 & 1.5 \\
\hline Electrode & & \\
\hline$\sigma^{\mathrm{eff}} / \mathrm{S} \mathrm{m}^{-1}$ & 0.6 & \\
\hline$D_{\mathrm{s}} / \mathrm{m}^{2} \mathrm{~s}^{-1}$ & $\approx 1.3 \cdot 10^{-15}$ (this work) & \\
\hline Kinetics & & \\
\hline$i_{00} / \mathrm{A} \mathrm{m}^{2.5} \mathrm{~mol}^{-1.5}$ & $3.34 \cdot 10^{-7}$ (this work) & - \\
\hline$U_{0} / \mathrm{V}$ & Eq. 7 (this work) & - \\
\hline$\alpha /-$ & 0.5 & - \\
\hline$c_{s}^{\max } / \mathrm{mol} \mathrm{m}^{-3}$ & 36027 (this work) & - \\
\hline Geometry & & \\
\hline Thickness / $\mu \mathrm{m}$ & 18 & 780 \\
\hline Area $/ \mathrm{cm}^{2}$ & 1.13 & 1.5394 \\
\hline$R_{\mathrm{p}} / \mu \mathrm{m}$ & 5.09 & - \\
\hline$\varepsilon_{\mathrm{e}} /-$ & 0.435 & 0.9 \\
\hline$\varepsilon_{\mathrm{NCM}} /-$ & 0.455 & - \\
\hline$\varepsilon_{\text {Filler }} /$ - & 0.058 & 0.1 \\
\hline$\varepsilon_{\mathrm{PVDF}} /-$ & 0.053 & - \\
\hline
\end{tabular}

Table 2: Simulation parameters of the P2D model determined on thin film cells. 